\title{
Venous thromboembolism risk factors and prophylaxis of elderly intensive care unit patients in a Chinese general hospital
}

\author{
Xiaolan Chen ${ }^{1 \#}$, Jiali Huang ${ }^{1 \#}$, Jingxuan Liu ${ }^{2}$, Hui Deng ${ }^{1}$, Lei Pan ${ }^{1}$ \\ ${ }^{1}$ Department of Respiratory and Critical Care Medicine, Beijing Shijitan Hospital, Capital Medical University, Beijing, China; ${ }^{2}$ Capital Medical \\ University, Beijing, China \\ Contributions: (I) Conception and design: X Chen, J Huang, L Pan; (II) Administrative support: J Liu, H Deng; (III) Provision of study materials or \\ patients: J Huang, J Liu; (IV) Collection and assembly of data: J Huang, J Liu; (V) Data analysis and interpretation: X Chen; (VI) Manuscript writing: \\ All authors; (VII) Final approval of manuscript: All authors. \\ \#These authors contributed equally to this work. \\ Correspondence to: Lei Pan, MD. Department of Respiratory and Critical Care Medicine, Beijing Shijitan Hospital, Capital Medical University, \\ Haidian, Beijing 100038, China. Email: panlei@bjsjth.cn.
}

Background To analyze the venous thromboembolism (VTE) risk factors and prophylaxis of elderly patients in the intensive care unit (ICU).

Methods: We retrospectively recruited 200 elderly patients with VTE and 234 non-VTE patients. VTE risk factors were got from data of all patients. The sensitivity and specificity of the Caprini scores and Padua scores were calculated. We use the receiver operating characteristic (ROC) curve and the area under the curve (AUC) to evaluate each score.

Results: We found that male sex, sepsis, being bedridden ( $>72$ hours), pneumonia, history of deep vein thrombosis (DVT), diabetes mellitus, coronary heart disease, heart failure, glucocorticoid treatment, $\mathrm{PaO}_{2}$, hemoglobin $(\mathrm{Hb})$, prothrombin time $(\mathrm{PT})$, and international normalized ratio (INR), D-dimer (D-D), mechanical ventilation, and continuous renal replacement therapy (CRRT) were significantly associated with VTE in elderly ICU patients $(\mathrm{P}<0.05)$. For elderly patients in the ICU, the predictive ability of Caprini risk assessment model was better than that of the Padua risk assessment model. Among the high VTE risk elderly patients, the number of patients receiving mechanical prophylaxis in the high bleeding-risk group was higher than that in the low bleeding-risk group $(\mathrm{P}<0.0001)$.

Conclusions: Elderly patients in the ICU have the highest risk of VTE and high bleeding risk; decisions concerning clinical prophylaxis should be made after appropriate information on the risk and benefit on an individual level is considered.

\begin{abstract}
Keywords: Venous thromboembolism (VTE); risk factors; prophylaxis; elderly intensive care unit (ICU); Chinese general hospital
\end{abstract}

Submitted Jan 28, 2021. Accepted for publication Mar 30, 2021.

doi: 10.21037/apm-21-464

View this article at: http://dx.doi.org/10.21037/apm-21-464

\section{Introduction}

Venous thromboembolism (VTE) includes deep vein thrombosis (DVT) and pulmonary thromboembolism (PE). The prevalence of VTE in medical inpatients is $12 \%$, the peak of VTE incidence is 65-75 years old, and the elderly are usually the patients at highest risk of VTE (1). VTE causes more than 100,000 deaths every year, with more than half of deaths occurring during hospitalization, especially in elderly patients. Approximately $18 \%$ to $65 \%$ of VTE-associated mortality has been reported as being preventable (2). Multimorbidity, which is defined as the co-occurrence of 2 or more chronic comorbidities is the common characteristic in the majority of elderly patients. We present the following article in accordance with the STARD reporting checklist 
(available at http://dx.doi.org/10.21037/apm-21-464).

\section{Methods}

\section{Patients}

We conducted a retrospective case-control study in which the data from 200 patients with VTE and 234 nonVTE patients were consecutively collected from January, 2019 to December 31, 2019 from the intensive care unit (ICU) in Beijing Shijitan Hospital. In the VTE group, the inclusion criteria were the following: $\geq 65$ years, $\geq 2$ days in the ICU, DVT diagnosed by lower extremity venous duplex compression ultrasonography and $\mathrm{PE}$ diagnosed by computed tomography (CT) angiography of the pulmonary artery or ventilation-perfusion imaging. The exclusion criteria were as follows: $<65$ years, DVT and PE diagnosed before admittance to the ICU or $<2$ days in ICU, and superficial vein thrombosis. This study was approved by the Medical ethics committee of Beijing Shijitan Hospital Affiliated to Capital Medical University (No. 2020-2). All procedures performed in this study involving human participants were in accordance with the Declaration of Helsinki (as revised in 2013). Individual consent for this retrospective analysis was waived.

\section{Study methods}

The clinical data, laboratory examination results, and treatment of the patients in the VTE group and non-VTE group admitted to the ICU in 2019 were obtained through the hospital's medical record system. The Caprini risk assessment model (RAM) and the Padua RAM were used for the 2 groups. The Caprini RAM includes 41 risk factors, with each factor being scored between 1 and 5 points and being classified into one of the following categories: low risk [0-1], moderate risk [2], high risk [3-4], or highest risk $[\geq 5]$. The Padua RAM includes 11 risk factors, with each factor being scored between 1 and 5 points and being classified into one of the following categories: low risk $(<4)$ or high risk ( $\geq 4)$. Two trained investigators were responsible for collecting data.

\section{Statistical analysis}

All data were analyzed by SPSS version 25 (IBM Corp., Armonk, NY, USA), and the count data are expressed as a percentage. $\chi^{2}$ test and Fisher's exact probability test were used for comparison between groups. The measurement data are expressed as $\bar{X} \pm$ S. If the distribution of data was normal, a parametric model was used to calculate the $\mathrm{P}$ value and confidence interval. For a nonnormal distribution of data, a nonparametric rank-sum test was used calculate the $\mathrm{P}$ value and confidence interval. We used logistic regression to analyze the risk factors in the VTE group. We drew receiver operating characteristic (ROC) curves and calculated the sensitivity, specificity and the value of the area under the curve (AUC).

\section{Results}

\section{Comparison of clinical characteristics between VTE and non VTE elderly ICU patients}

The number of male patients in the VTE group was 152 (76\%), which was greater than that in the $128(54.7 \%)$ in the non-VTE group $(\mathrm{P}<0.05)$. We found that in the VTE group, the proportion of patients suffering from sepsis ( $<1$ month), bed rest ( $>72$ hours), history of DVT were higher than that in the non-VTE group $(\mathrm{P}<0.05)$. However, the number of patients receiving glucocorticoid treatment in the non-VTE group was more than that in the VTE group $(\mathrm{P}<0.05)$. In terms of laboratory examination, levels of $\mathrm{PaO}_{2}$, hemoglobin $(\mathrm{Hb})$, prothrombin time $(\mathrm{PT})$, and international normalized ratio (INR) in the VTE group were lower than those in the non-VTE group $(\mathrm{P}<0.05)$; however, D-dimer (D-D), creatinine (Cr), APACHE II (Acute Physiology And Chronic Health Evaluation II), mechanical ventilation, hemofiltration, nutritional risk score, Caprini score, and Padua score in the VTE group were much higher than those in the non-VTE group $(\mathrm{P}<0.05)$. The number of elderly patients receiving mechanical prevention and anticoagulation therapy in the non-VTE group was greater than that in the VTE group $(\mathrm{P}<0.05$; Table 1).

\section{Risk factors of VTE in elderly ICU patients}

Before the logistic regression analysis, the data were standardized. The results showed that male sex, sepsis, being bedridden ( $>72$ hours), history of DVT, glucocorticoid treatment, $\mathrm{PaO}_{2}, \mathrm{Hb}, \mathrm{PT}$, INR, D-D, mechanical ventilation, and continuous renal replacement therapy (CRRT), were significantly associated with VTE in 
Table 1 Characteristics of elderly ICU patients in the ICU in the VTE and non-VTE groups

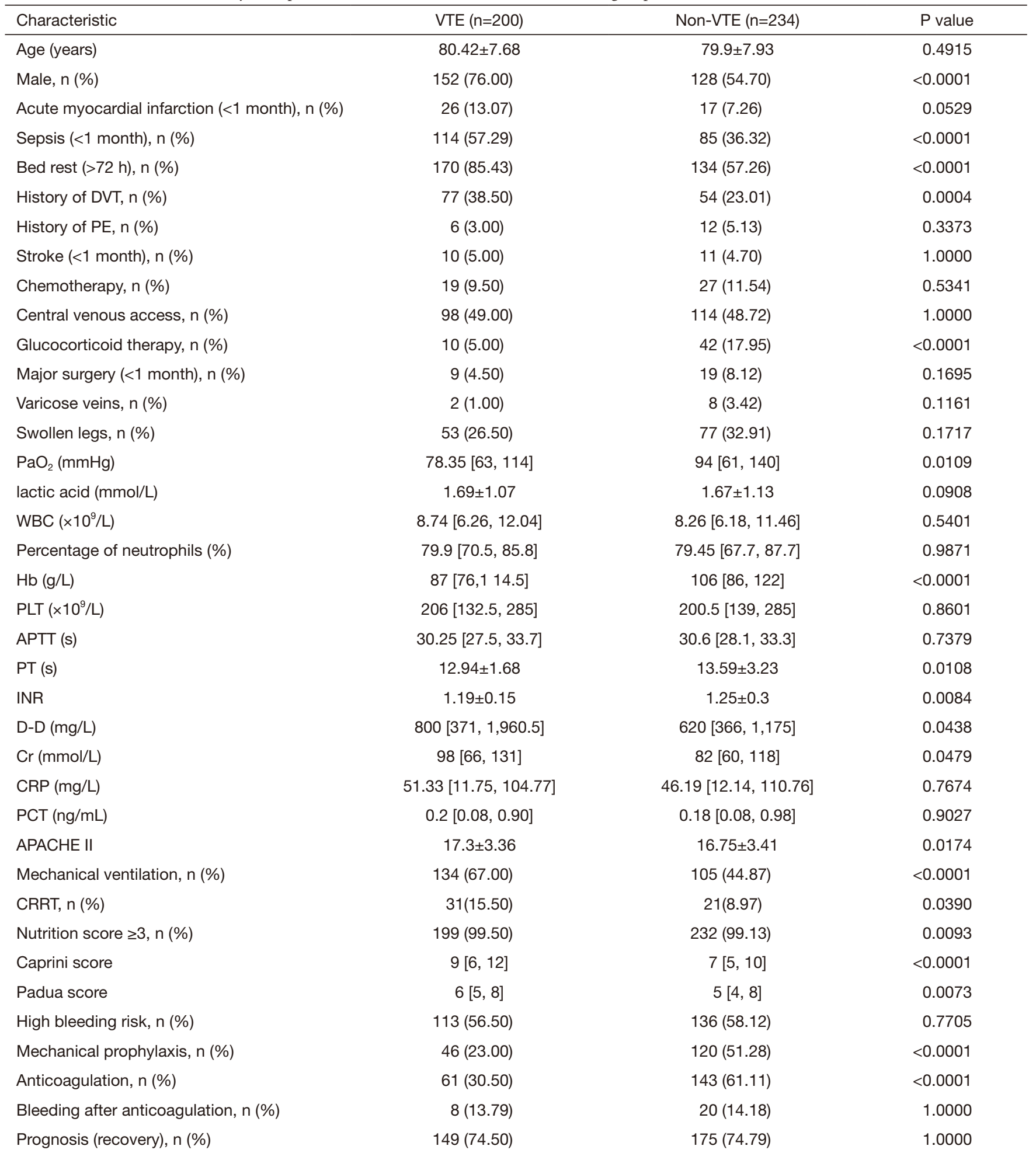

ICU, intensive care unit; VTE, venous thromboembolism; DVT, deep vein thrombosis; PE, pulmonary thromboembolism; WBC, leukocyte; $\mathrm{Hb}$, hemoglobin; PLT, blood platelet; APTT, activated partial thromboplastin time; PT, prothrombin time; INR, international normalized ratio; D-D, D-dimer; Cr, creatinine; CRP, C-reactive protein; PCT, procalcitonin; APACHE II, Acute Physiology and Chronic Health Evaluation II; CRRT, continuous renal replacement therapy. 
Table 2 VTE risk factors of elderly patients in the ICU

\begin{tabular}{|c|c|c|c|c|}
\hline Characteristic & OR & $\mathrm{Cl}(2.5 \%)$ & $\mathrm{Cl}(97.5 \%)$ & $P$ value \\
\hline Sepsis (<1 month) (\%) & 2.87 & 1.90 & 4.34 & $<0.001$ \\
\hline Bed rest (>72 h) (\%) & 5.36 & 3.25 & 8.85 & $<0.001$ \\
\hline History of DVT (\%) & 0.47 & 0.30 & 0.73 & 0.001 \\
\hline $\mathrm{PaO}_{2}(\mathrm{mmHg})$ & 0.99 & 0.99 & 1.00 & $<0.001$ \\
\hline $\mathrm{Hb}(\mathrm{g} / \mathrm{L})$ & 1.02 & 1.01 & 1.03 & $<0.001$ \\
\hline PT (s) & 1.13 & 1.03 & 1.25 & 0.013 \\
\hline INR & 3.97 & 1.40 & 11.27 & 0.010 \\
\hline APACHE II & 0.94 & 0.89 & 1.00 & 0.055 \\
\hline Mechanical ventilation (\%) & 2.37 & 1.59 & 3.54 & $<0.001$ \\
\hline CRRT (\%) & 2.09 & 1.13 & 3.86 & $<0.001$ \\
\hline Nutrition score $\geq 3$ (\%) & 0.52 & 0.04 & 6.04 & 0.599 \\
\hline
\end{tabular}

VTE, venous thromboembolism; ICU, intensive care unit; OR, odds ratio; Cl, confidence interval; DVT, deep-vein thrombosis; Hb, hemoglobin; PT, prothrombin time; INR, international normalized ratio; D-D, D-dimer; Cr, creatinine; APACHE II, Acute Physiology and Chronic Health Evaluation II; CRRT, continuous renal replacement therapy.

Table 3 Comparison of elderly ICU patients in the ICU in the VTE and non-VTE groups with different disease

\begin{tabular}{lccc}
\hline Disease & VTE $(\mathrm{n}=200)$ & Non-VTE $(\mathrm{n}=234)$ & $\mathrm{P}$ value \\
\hline Currently pneumonia, $\mathrm{n}(\%)$ & $172(86.87)$ & $181(77.35)$ & 0.0124 \\
COPD, $\mathrm{n}(\%)$ & $28(14.07)$ & $37(15.81)$ & 0.6860 \\
Hypertension, $\mathrm{n}(\%)$ & $124(62.31)$ & $152(64.96)$ & 0.6163 \\
Cancer, $\mathrm{n}(\%)$ & $65(32.66)$ & $54(23.08)$ & 0.0307 \\
Diabetes, $\mathrm{n}(\%)$ & $128(64.00)$ & $107(45.73)$ & 0.0002 \\
Coronary heart disease, $\mathrm{n}(\%)$ & $174(87.00)$ & $164(70.09)$ & $<0.0001$ \\
Cardiac failure, $\mathrm{n}(\%)$ & $156(78.00)$ & $147(62.82)$ & 0.0008 \\
\hline
\end{tabular}

VTE, venous thromboembolism; COPD, chronic obstructive pulmonary disease;

elderly ICU patients $(\mathrm{P}<0.05$; Table 2).

\section{Comparison between VTE and non VTE elderly ICU patients with different disease}

We found in the VTE group, pneumonia, and incidence of tumor was higher than that in the non-VTE group $(\mathrm{P}<0.05)$. The number of patients in the VTE group with diabetes mellitus, coronary heart disease, and heart failure was greater than that in the non-VTE group $(\mathrm{P}<0.05$; Table 3). We documented that pneumonia, diabetes mellitus, coronary heart disease, heart failure were significantly associated with 
Table 4 VTE risk factors of elderly patients with different disease in the ICU

\begin{tabular}{|c|c|c|c|c|}
\hline Disease & OR & $\mathrm{Cl}(2.5 \%)$ & $\mathrm{Cl}(97.5 \%)$ & $P$ value \\
\hline Cancer (\%) & 1.45 & 0.94 & 2.24 & 0.095 \\
\hline Diabetes (\%) & 2.37 & 1.59 & 3.56 & $<0.001$ \\
\hline Coronary heart disease (\%) & 3.08 & 1.84 & 5.15 & $<0.001$ \\
\hline
\end{tabular}

OR, odds ratio; $\mathrm{Cl}$, confidence interval.

Table 5 The comparison between Caprini and Padua RAMs in elderly ICU patients

\begin{tabular}{lccc}
\hline Measure & Caprini & Padua & P value \\
\hline Sensitivity & 0.687 & 0.350 & $<0.0001$ \\
Specificity & 0.540 & 0.815 & $<0.0001$ \\
AUC & 0.6303 & 0.5719 & 0.0495
\end{tabular}

RAM, risk assessment model; ICU, intensive care unit; AUC, area under the curve.

VTE in elderly ICU patients $(\mathrm{P}<0.05$; Table 4).

\section{Comparing the Padua and Caprini RAMs in elderly ICU patients}

The sensitivity of the Caprini RAM (0.687) was higher than that of the Padua RAM $(0.35)(\mathrm{P}<0.0001)$, but compared with the Caprini RAM (0.54), the specificity of the Padua RAM $(0.815)$ was significantly higher $(\mathrm{P}<0.0001)$ (Table 5). The AUC value of Caprini RAM was significantly greater than that of the Padua RAM (0.6303 vs. 0.5719, $\mathrm{P}<0.05$ ). For elderly ICU patients, the Caprini RAM has a higher sensitivity but a lower specificity than the Padua RAM. Overall, the predictive ability of the Caprini RAM was better than that of the Padua RAM (Figure 1).

\section{Comparing prophylaxis in bigh VTE risk patients with bleeding risk among elderly ICU patients}

Among the elderly high VTE risk patients, mechanical prophylaxis alone was much more common in high bleeding-risk patients than in low bleeding-risk patients (27.35\% vs. $13.14 \%, \mathrm{P}<0.0001)$. Another 2 prophylaxis types, including anticoagulation prophylaxis alone and mechanical + anticoagulation, showed no significant difference between the different bleeding risks $(\mathrm{P}>0.05$;

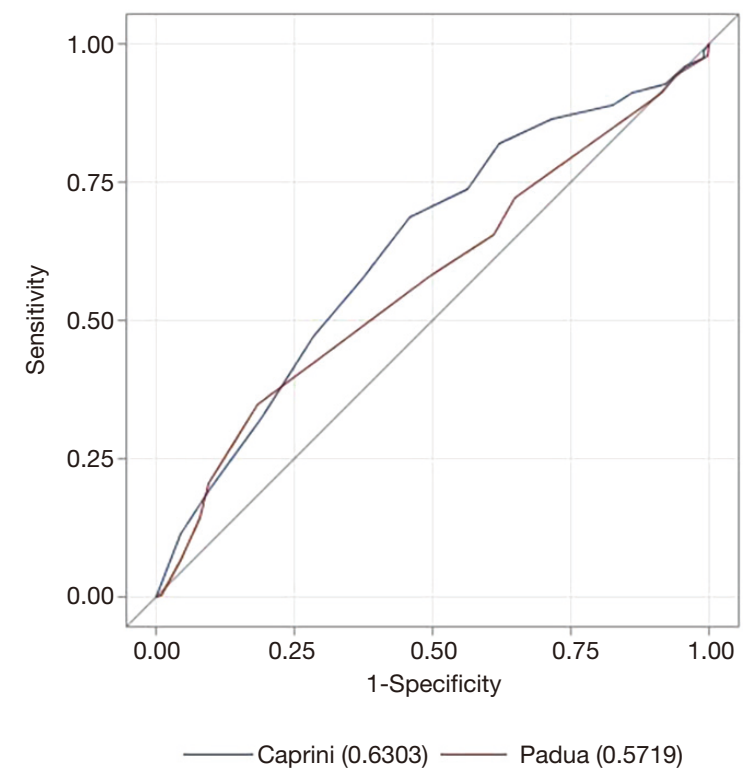

Figure 1 The performance of the Caprini and Padua RAMs in elderly ICU patients. RAM, risk assessment model; ICU, intensive care unit.

Table 6).

\section{Discussion}

Many large multicenter studies exclude elderly patients due to the multiple diseases present in many elderly patients. At present, there are few studies on VTE in elderly patients, especially in elderly ICU patients. Engbers $e t a l$. found the annual incidence of VTE in elderly patients to be nearly 8,000 , which is 8 times higher than that in patients under 50 years old (3). It is well known that elderly patients often have multiple diseases. Pleuritic chest pain, hemoptysis, tachycardia, swollen extremities, and difficulty walking, which are typical signs and symptoms of VTE, are more 
Table 6 Prophylaxis types and bleeding risk in elderly ICU patients with high VTE risk

\begin{tabular}{lccc}
\hline Bleeding risk & $\begin{array}{c}\text { Mechanical prophylaxis alone, } \\
\mathrm{n}(\%)\end{array}$ & $\begin{array}{c}\text { Anticoagulation prophylaxis alone, } \\
\mathrm{n}(\%)\end{array}$ & $\begin{array}{c}\text { Mechanical + anticoagulation, } \\
\mathrm{n}(\%)\end{array}$ \\
\hline High bleeding risk $(\mathrm{n}=245)$ & $67(27.35)$ & $33(13.47)$ & $20(8.16)$ \\
Low bleeding risk $(\mathrm{n}=175)$ & $23(13.14)$ & $22(12.57)$ & $22(12.57)$ \\
$\chi^{2}$ & 12.233 & 0.072 & 2.204 \\
$\mathrm{P}$ value & $<0.0001$ & 0.454 & 0.094 \\
\hline
\end{tabular}

ICU, intensive care unit; VTE, venous thromboembolism.

prevalent in elderly patients than in younger patients. A few researchers have reported that the prevalence of asymptomatic DVT is $17.8 \%$ among patients over 80 years old, and for ICU patients the prevalence of DVT is as high as 33\% (4-6). A large prospective multicenter Chinese trial confirmed that the prevalence of VTE during ICU stay was about $7.3 \%$ (7). Alessandro summarized some studies of COVID-19 and VTE, found that the incidence of VTE was about $30 \%$ in hospitalized COVID-19 patients, the incidence of DVT alone and PE alone was about $20 \%$ and $18 \%$ respectively (8). Huang et al. reported the incidence of VTE sharply increased among those older than 75 years old (9). Zhang et al. confirmed that the incidence of VTE within 7 days of ICU admission was as high as $4.45 \%$ (10).

Our study found that the proportion of male patients in the VTE group was much higher than that in the nonVTE group, while sepsis, being bedridden ( $>72$ hours), pneumonia, and presence of tumor were also significantly higher; with sepsis, being bedridden ( $>72$ hours), and pneumonia were associated with VTE. Hypercoagulability and blood stasis are the basis of thrombosis (11). Blood clot occurs frequently due to increased coagulation factor activity in elderly patients (12). Furthermore, interleukin 6 (IL-6) cytokine is secreted by monocyte macrophages and $\mathrm{T}$ lymphocytes after infection. The high concentration of IL- 6 can damage vascular endothelial cells, promote immune adhesion and micro thrombosis, inhibit endothelial repair, and damage blood vessels (13). In the addition, a large number of other inflammatory cell infiltrates release inflammatory factors and inflammatory mediators, resulting in systemic inflammatory response syndrome, leading to lung tissue damage, which can initiate endogenous coagulation and form microthrombosis in the blood circulation (14). Kaplan et al. demonstrated that the incidence of VTE in patients with sepsis is higher than in those without sepsis; it has also been found that the deposition of platelet-fibrin thrombi and the development of disseminated intravascular coagulation are dysregulated in these patients (15).

In the VTE group, there were more patients with a history of DVT, diabetes, coronary heart disease, and heart failure, which were significantly correlated with the incidence of VTE in elderly ICU patients. Wang et al. showed that the proportion of hypertension, active malignant tumor/ chemotherapy, diabetes, coronary heart disease, heart and/ or respiratory failure, acute myocardial infarction, and/ or ischemic stroke to be significantly increased in elderly patients with VTE in the ICU (16). Our study was consistent with the above findings. In terms of laboratory examination, $\mathrm{PaO}_{2}, \mathrm{Hb}, \mathrm{PT}$, and INR in the VTE group were lower than those in the non-VTE group $(\mathrm{P}<0.05)$, while $\mathrm{PaO}_{2}, \mathrm{Hb}, \mathrm{PT}$, and INR were significantly correlated with the occurrence of VTE. Wang et al. reported that $\mathrm{PaO}_{2}$ level is an important factor affecting the occurrence of VTE in elderly acute exacerbation of chronic obstructive pulmonary disease (AECOPD) patients (17). D-D, Cr, APACHE-II, mechanical ventilation, hemofiltration, nutritional risk score, Caprini score, and Padua score were significantly higher in the VTE group than in the non-VTE group. D-D, mechanical ventilation, and CRRT were important risk factors for VTE in elderly ICU patients. Fu et al. found D-D to be an independent factor of VTE in ICU patients. Laboratory examinations and Caprini risk assessment scale can increase the predictive ability of VTE in ICU patients (18). However, the specificity of D-D is poor, and it increases in the cases of tumor, infection, and trauma. Multiple diseases coexist in elderly patients. For physicians, it is necessary to dynamically observe the level of D-D. Liu et al. found that D-D was significantly associated with VTE in elderly AECOPD patients (19). Li et al. confirmed that, with the increase of APACHE II score, the mortality rate gradually increases: when the score is $\geq 25$, the mortality rate increases significantly; when the score is $\geq 35$, the mortality rate increases to $100 \%$. The actual mortality rate is significantly 
and positively correlated with APACHE II score (20). Studies by An et al. evaluated the APACHE II score of a death group 24 hours after admission and found that their score was higher than that of the survival group. It has also been found that the higher the Nutrition Risk Screening 2002 (NRS-2002) value is, the worse the prognosis (21). Another retrospective study by Adimi Naghan et al. demonstrated that if the APACHE II score is greater than 17.6, most cases develop VTE in the first 2 weeks of ICU stay (22). Development of acute respiratory distress syndrome (ARDS) and higher positive end-expiratory pressure (PEEP) were associated with an increased risk for development of VTE (23). Prabhakaran et al. demonstrated that increased ventilator days were also associated with higher risk of developing VTE (24).

In our study, the number of elderly patients receiving mechanical prophylaxis and anticoagulation therapy in the non-VTE group was significantly higher than that in the VTE group. The 9th edition of US guidelines on the prevention of venous thrombosis pointed out that prevention of VTE should be given to elderly patients at high risk of VTE (25). High risk factors such as age, bedridden, coexistence of multiple diseases and central venous catheterization lead elderly ICU patients to be high or highest-risk group of VTE. If there is no contraindication, low dose unfractionated heparin (LDUH) (5,000 u subcutaneous injection, twice a day) or low molecular weight heparin (LMWH) (such as enoxaparin $40 \mathrm{mg}$ subcutaneous injection, once a day; dalteparin $5,000 \mathrm{u}$ subcutaneous injection, once a day) should be recommended for high-risk patients in ICU to prevent the occurrence of VTE. And mechanical prophylaxis should be combined with chemoprophylaxis. For patients with high risk of bleeding, graduated compression stockings (GCS) and (or) intermittent pneumatic compression (IPC) were used to prevent thrombosis until the risk of bleeding was reduced, and then chemoprophylaxis should be prescribed or both were used in combination. ICU patients should be evaluated dynamically according to the development of the disease (26). García-Olivares and other researchers discovered that establishing a prophylaxis protocol in the ICU can reduce the incidence of VTE from $11.9 \%$ to $4.5 \%$ $(27,28)$. In regard to mechanical prophylaxis, Vignon et al. showed that, compared with those receiving GCS alone, there was no difference in the incidence of VTE in those who received IPC together with GCS (29). For nursing strategy in the our intensive care unit, the patients without consciousness will be given GCS and IPC, the patients with consciousness will be given health education of VTE besides the above mechanical prophylaxis.

There is no specific VTE risk assessment model for elderly patients in ICU. We used the Caprini RAM and Padua RAM which were used commonly in clinic. And found that the sensitivity of the Caprini RAM was higher than that of the Padua RAM in elderly ICU inpatients, and that the Caprini RAM was a good predictor of VTE risk in elderly ICU patients. Our previous research discovered similar conclusions across different departments. We compared the sensitivity and specificity of the 2 scales in VTE risk patients between different departments (internal $v s$. surgical, oncological $v s$. nononcological) in a general hospital. We found that the Caprini RAM had a higher sensitivity but a lower specificity than the Padua RAM and had better VTE predictive ability among hospitalized Chinese patients (30).

Most of the elderly ICU patients are at high-risk of VTE. Furthermore, due to the coexistence of multiple diseases in elderly patients, the interaction between drugs and the decline of liver and kidney function, the risk of bleeding is significantly increased. Parikh et al. found that only $20.2 \%$ of medical ICU patients received VTE prophylaxis which were recommended by American College of Chest Physicians (ACCP) (31). In this study, we found that mechanical prophylaxis was the main prophylaxis method for high bleeding-risk patients, which was consistent with the recommendations of the Caprini RAM (32). Arabi et al. found that only using IPC was associated with a significantly decreased incidence of VTE. The ACCP recommend that IPC can be used for patients with contraindication to drug thromboprophylaxis (33). In addition, our study found no significant difference between the VTE group and nonVTE group in bleeding after anticoagulation. Another trial suggested that patients older than 75 years who were treated with novel oral anticoagulants (NOACs) had a greater reduction in the risk of major bleeding than those treated with vitamin $\mathrm{K}$ antagonists (VKAs) (34). In the recent multiple centers research including 41 tertiary hospitals in China, Zhou found that $85.4 \%$ ICUs completed VTE risk assessment within 24 hours after admission. $71.4 \%$ ICUs used the Caprini score to assess VTE risk. $97.6 \%$ of ICUs used IPC as mechanical prophylaxis. LMWH was the initial chemoprophylaxis in ICUs (35).

Our study had a few limitations which should be mentioned. First, the discharged patients were not followed up, we will follow-up the discharged patients in our further research to evaluate the prognosis of the elderly 
ICU patients. Second, because there was no similar study in the past, we used retrospective study to explore. In the future, large cohort study will be needed to verify. VTE is a preventable death related to hospitalization, and it is also one of the main causes of medical complications. With the aging global population, elderly patients are becoming a high-risk VTE group due to their age, fragility, frequent bed rest, presence of other diseases, etc., and the elderly ICU patients are the key population for VTE prevention and control. However, balancing the benefit and risk of treatment requires us to dynamically assess the risk of VTE and bleeding in elderly patients. In the next phase of our research, we plan to conduct a prospective study on the individualized comprehensive management of VTE in elderly ICU patients. Combined with biomarkers, imaging, VTE risk assessment scale, and bleeding risk assessment scale, a precise treatment plan for elderly ICU patients can be formulated.

\section{Acknowledgments}

The authors are grateful for the patients at Beijing Shijitan Hospital.

Funding: The authors disclosed receipt of the following financial support for the research, authorship, and/or publication of this article: National Multidisciplinary Cooperative Diagnosis and Treatment Capacity Project for Major Diseases: Comprehensive Treatment and Management of Critically Ill Elderly Inpatients (No. 2019.YLFW); Beijing Municipal Science \& Technology Commission Research Project (No. 2018-A20).

\section{Footnote}

Reporting Checklist: The authors have completed the STARD reporting checklist. Available at http://dx.doi.org/10.21037/ apm-21-464

Data Sharing Statement: Available at http://dx.doi. org/10.21037/apm-21-464

Conflicts of Interest: All authors have completed the ICMJE uniform disclosure form (available at http://dx.doi. org/10.21037/apm-21-464). The authors have no conflicts of interest to declare.

Ethical Statement: The authors are accountable for all aspects of the work in ensuring that questions related to the accuracy or integrity of any part of the work are appropriately investigated and resolved. This study was approved by the Medical ethics committee of Beijing Shijitan Hospital Affiliated to Capital Medical University (No. 2020-2). All procedures performed in this study involving human participants were in accordance with the Declaration of Helsinki (as revised in 2013). Individual consent for this retrospective analysis was waived.

Open Access Statement: This is an Open Access article distributed in accordance with the Creative Commons Attribution-NonCommercial-NoDerivs 4.0 International License (CC BY-NC-ND 4.0), which permits the noncommercial replication and distribution of the article with the strict proviso that no changes or edits are made and the original work is properly cited (including links to both the formal publication through the relevant DOI and the license). See: https://creativecommons.org/licenses/by-nc-nd/4.0/.

\section{References}

1. Chen XL, Wang Y, Pan L. Validity of caprini risk assessment scale for assessing risk of deep venous thrombosis in elderly patients with severe pneumonia. Beijing Med 2016;38:989-93.

2. Voils SA, Carlson A. Association of deep-vein thrombosis (DVT) with missed doses of prophylactic antithrombotic medications in ICU patients: a case-control study. Ann Pharmacother 2014;48:1294-7.

3. Engbers MJ, van Hylckama Vlieg A, Rosendaal FR. Venous thrombosis in the elderly:incidence, risk factors and risk groups. J Thromb Haemost 2010;8:2105-112.

4. Lawall H, Oberacker R, Zemmrich C, et al. Prevalence of deep vein thrombosis in acutely admitted ambulatory non-surgical intensive care unit patients. BMC Res Notes 2014;7:431.

5. Minet $\mathrm{C}$, Potton L, Bonadona A, et al. Venous thromboembolism in the ICU: main characteristics, diagnosis and thromboprophylaxis. Crit Care 2015;19:287.

6. Boddi M, Peris A. Deep Vein Thrombosis in Intensive Care. Adv Exp Med Biol 2017;906:167-81.

7. Li XY, Fan J, Cheng YQ, et al. Incidence and prevention of venous thromboembolism in acutely ill hospitalized elderly Chinese. Chin Med J 2011;124:335-40.

8. Di Minno A, Ambrosino P, Calcaterra I, et al. COVID-19 and Venous Thromboembolism: A Metaanalysis of Literature Studies. Semin Thromb Hemost 2020;46:763-71. 
9. Huang D, Chan PH, She HL, et al. Secular trends and etiologies of venous thromboembolism in Chinese from 2004 to 2016. Thromb Res 2018;166:80-5.

10. Zhang C, Zhang Z, Mi J, et al. The cumulative venous thromboembolism incidence and risk factors in intensive care patients receiving the guidelinerecommended thromboprophylaxis. Medicine (Baltimore) 2019;98:e15833.

11. Alhassan S, Pelinescu A, Gandhi V, et al. Clinical presentation and risk factors of venous thromboembolic disease. Crit Care Nurs Q 2017;40:201-9.

12. Liss D. Hypercoagulability and adrenal dysfunction in critical care. Vet Technician 2010;31:E1-E3.

13. Bai JW, Gao BL, Xu JF, et al. Mechanisms of pulmonary embolism and/ or deep vein thrombosis secondary to chronic obstructive pulmonary disease exacerbation in elders. Chin J Gen Pract 2014;1:448-51.

14. Wei X, Xu ML, Ning B. Analysis of the Death of Risk Factors in Elderly Patients with Critical Disease, Med J Air Force 2011;27:92-5.

15. Kaplan D, Casper TC, Elliott CG, et al. VTE Incidence and Risk Factors in Patients With Severe Sepsis and Septic Shock. Chest 2015;148:1224-30.

16. Wang X, Liu SH, Hong XY, et al. Epidemiological survey of venous thromboembolism in the elderly in-patients in Internal Medicine Department of Peking Union Medical College Hospital. Chin J Mult Organ Dis Elderly 2018;17:491-5.

17. Wang $W$. Incidence and influencing factors of venous thromboembolism in elderly patients with acute exacerbation of COPD. Chin Med Analysis 2018;25:40$41+45$.

18. Fu Y, Liu Y, Chen S, et al. The combination of Caprini risk assessment scale and thrombotic biomarkers to evaluate the risk of venous thromboembolism in critically ill patients. Medicine (Baltimore) 2018;97:e13232.

19. Liu X, Zhao T, Bi QJ, et al. Characteristics and risk factors of deep venous thrombosis in elderly patients with chronic obstructive pulmonary disease in emergency intensive care unit. Chin J Geriatr 2019;39:3154-6.

20. Li J, Hui J, Luo JH. Application value of APACHE II for elderly patients with severe disease. World J Integr Tradit West Med 2012;21:695-696,699.

21. An ZH, Wang DH, Zhang J, et al. The relationship among Nutrition Risk Screening 2002, APACHE II and the prognosis of critically ill elderly patients. J Inter Inten Med 2014;20:235-236+243.

22. Adimi Naghan P, Malekmohammad M, Jamaati H, et al.
Venous thromboembolism in medical critically ill patients: prevalence and incidence. Acta Med Iran 2013;51:168-71.

23. Hanify JM, Dupree LH, Johnson DW, et al. Failure of chemical thromboprophylaxis in critically ill medical and surgical patients with sepsis. J Crit Care 2017;37:206-10.

24. Prabhakaran K, Gogna S, Lombardo G, et al. Venous Thromboembolism in Geriatric Trauma PatientsRisk Factors and Associated Outcomes. J Surg Res 2020;254:327-33.

25. Respiratory Branch of Chinese Medical Association. Chinese expert consensus on prevention of venous thromboembolism in medical inpatients (2015). Chin J Geriatr 2015;34:345-52.

26. Pai M, Adhikari NKJ, Ostermann M, et al. PROTECT (Prophylaxis for Thromboembolism in Critical Care Trial) Investigators. Low-molecular-weight heparin venous thromboprophylaxis in critically ill patients with renal dysfunction: A subgroup analysis of the PROTECT trial. PLoS One 2018;13:e0198285.

27. García-Olivares P, Guerrero JE, Keough E, et al. PROFETEV study investigators. Clinical factors associated with inappropriate prophylaxis of venous thromboembolic disease in critically ill patients. A single day cross-sectional study. Thromb Res 2016;143:111-7.

28. Gould MK, Garcia DA, Wren SM, et al. Prevention of VTE in nonorthopedic surgical patients: Antithrombotic Therapy and Prevention of Thrombosis, 9th ed: American College of Chest Physicians Evidence-Based Clinical Practice Guidelines. Chest 2012;141:e227S-77S.

29. Vignon P, Dequin PF, Renault A, et al. Clinical Research in Intensive Care and Sepsis Group (CRICS Group). Intermittent pneumatic compression to prevent venous thromboembolism in patients with high risk of bleeding hospitalized in intensive care units: the CIREA1 randomized trial. Intensive Care Med 2013;39:872-80.

30. Chen X, Pan L, Deng H, et al. Risk Assessment in Chinese Hospitalized Patients Comparing the Padua and Caprini Scoring Algorithms. Clin Appl Thromb Hemost 2018;24:127S-35S.

31. Parikh KC, Oh D, Sittipunt C, et al. Venous thromboembolism prophylaxis in medical ICU patients in Asia (VOICE Asia): a multicenter, observational, crosssectional study. Thromb Res 2012;129:e152-8.

32. Caprini JA. Individual risk assessment is the best strategy for thromboembolic prophylaxis. Dis Mon 2010;56:552-9.

33. Arabi YM, Khedr M, Dara SI, et al. Use of intermittent pneumatic compression and not graduated compression stockings is associated with lower incident VTE in 
critically ill patients: a multiple propensity scores adjusted analysis. Chest 2013;144:152-9.

34. van Es N, Coppens M, Schulman S, et al. Direct oral anticoagulants compared with vitamin $\mathrm{K}$ antagonists for acute venous thromboembolism: evidence from phase 3 trials. Blood 2014;124:1968-75.

Cite this article as: Chen X, Huang J, Liu J, Deng H, Pan L. Venous thromboembolism risk factors and prophylaxis of elderly intensive care unit patients in a Chinese general hospital. Ann Palliat Med 2021;10(4):4453-4462. doi: 10.21037/apm-21-464
35. Zhou J, Li L, Zhen JH, et al. Current prophylaxis and treatment of venous thromboembolism in intensive care units of 41 tertiary hospitals of Zhejiang Province. Zhonghua Nei Ke Za Zhi 2021;60:139-42.

(English Language Editor: J. Gray) 